\title{
Modelling the elastic response of a polyurethane open cell foam based on a minimal surface energy approach
}

\author{
B. Buffel ${ }^{1}$, F. Desplentere ${ }^{1}, \mathrm{~K}$. Bracke $^{2} \&$ I. Verpoest ${ }^{3}$ \\ ${ }^{1}$ KHBO Expertisecentrum Kunststoffen, KULeuven-KHBO, Belgium \\ ${ }^{2}$ Recticel International Development Centre, Recticel nv, Belgium \\ ${ }^{3}$ Department of Metallurgy and Materials Engineering, \\ KULeuven, Belgium
}

\begin{abstract}
Within the present study the elastic response of a flexible open cell polyurethane foam was studied by means of experimental compression tests and finite element (FE) modelling. The compression tests revealed a pronounced sample size effect which was taken into account using an analytical model. In order to eliminate the sample size and damage effects, a minimal sample size of at least 50 times the cell size was necessary in the case of the flexible foam. Surface evolver software was used to model the open cell foam structures. The FE unit cells are based on the well-known Kelvin cell and the more complex Weaire-Phelan cell topology. In both cases the cross sectional shape of the cell edges was completely determined by the minimisation of the surface energy. The thus build FE-models possess a good resemblance to real open cell foam structures. The influence of relative density and shape anisotropy on the elastic properties of the cellular structures was analysed using the FE-models.
\end{abstract}

Keywords: open cell foam, Kelvin cell, Weaire-Phelan cell, surface evolver.

\section{Introduction}

Polyurethane foams exist in a wide variety of properties ranging from flexible and open celled to rigid with almost $100 \%$ closed cells. Therefore this material can be found in multiple applications such as thermal insulation in house building, cushioning in furniture or automotive seating, spacer material in 
sandwich structures and filtration parts in a vacuum cleaner (Randall and Lee [1]). The generation of the cellular structure in polyurethane foams is dominated by surface tension which distinguishes them from other foamed thermoplastics (Mills [2]). Because of these two reasons a lot of research has been done on the morphology and mechanical behaviour of foams in general and open cell polyurethane foams in particular.

The morphology of foam is characterised by the number and type of cell faces and the cross sectional area, shape and length of the cell edges. These characteristics were first quantified by the observations of (Matzke [3]) and were confirmed using (electron) microscopy (Gong et al. [4], Mills [5]) and CTimaging more recently (Jang et al. [6], Wismans et al. [7]). In general, a cell with 14 faces and a face with 5 edges proved to be the most abundant.

Another approach to study cellular materials is the search for a unit cell to divide space with a minimal surface energy. The most well-known solution for this problem is the 14 sided Kelvin cell. In contrast to experimental observations this unit cell consists of six square and eight hexagonal faces and possesses no pentagonal faces. This shortcoming is met by the Weaire-Phelan (Phelan et al. [8]) and P42 (Gabbrielli [9]) structures by making use of respectively 2 and 4 different volumes in one unit cell. This increased complexity of the unit cells produces a lower surface energy and better correspondence with experimental foam morphology compared to the common Kelvin cell. Within this research the Kelvin and Weaire-Phelan cell were selected to model the open cell foam structure.

Based on the bending of the cell edges of a rudimental cubic unit cell Gibson and Ashby developed a power law relationship between the stiffness and density of a cellular structure (Gibson and Ashby [10]). This model was later confirmed by applying its principles on the Kelvin cell geometry (Warren and Kraynik [11]). More detailed models including cell shape anisotropy and cell edges with a plateau border shaped cross section were developed by Gong et al. [4] and Jang et al. [6]. Surface evolver software was used by Jang et al. [6] and Mills [5] in order to build foam models with a minimised surface energy of the cell edges to obtain a remarkable resemblance to real foam structures. The most recent developed is the use of computed tomography images as a source for foam models (Wismans et al. [7]).

This study focuses on the development of FE-models of the Kelvin and Weaire-Phelan unit cell to determine the elastic response of open cell polyurethane foams. Surface evolver software was used to minimise the surface energy of the cell edges and produce FE-models which imitate real foams as good as possible. Experimental compression tests were performed to determine the stiffness of the open cell foam and validate the FE-models.

\section{Foam morphology}

The morphology of two types of commercially available open cell polyurethane foam was investigated. Both foam types were industrially produced and possessed an almost identical density with a maximum difference of $7 \%$. The 
cell size of the foams was determined by means of optical microscopy and was found to be $0,885 \mathrm{~mm}$ and $2,621 \mathrm{~mm}$. In order to analyse the number of faces per cell and number of edges per face, 10 single cells were manually cut out and analysed. Figures 1 and 2 presents the results of these observations and compares them to literature values and possible unit cells for foam modelling.

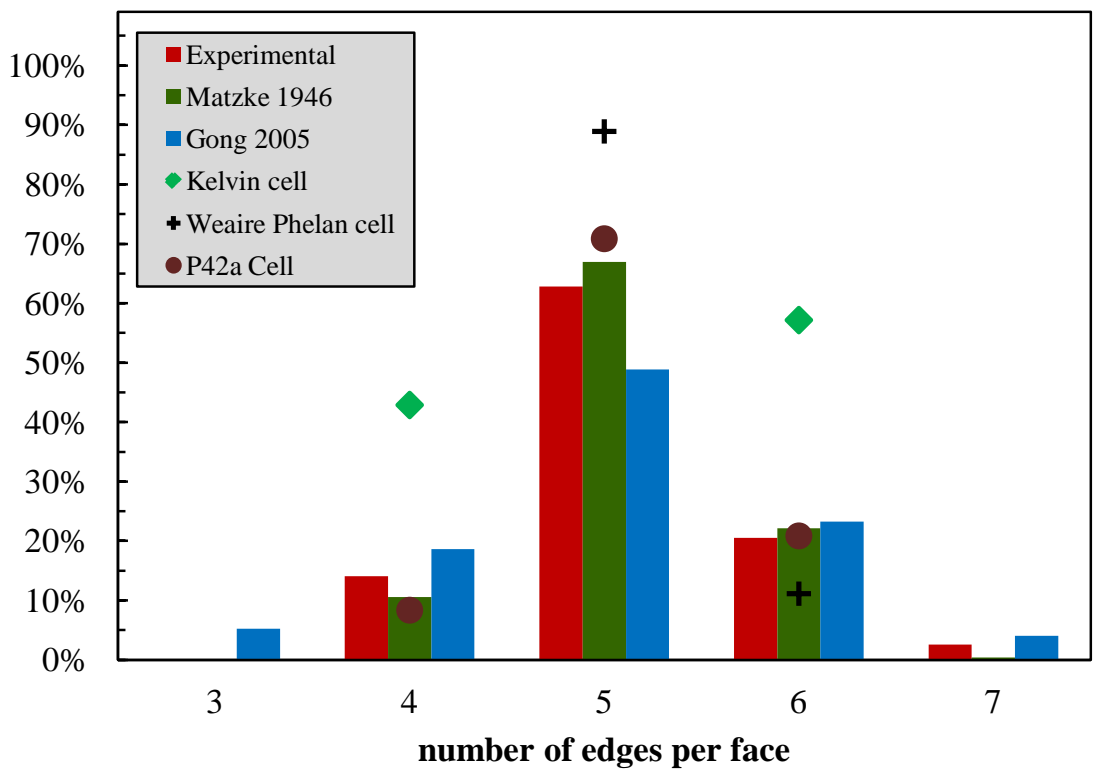

Figure 1: Distribution of the number of edges per face experimentally counted on 10 different single cell samples. Data from literature observations and 3 different unit cells are added to the figure as a comparison.

The experimentally observed morphology of the polyurethane foam is in excellent agreement with the values from literature. When the possible unit cells are compared to the experimental data, the P42a cell represents the real foam morphology the best. However, the FE-modelling was based on the more common Kelvin Cell and the Weaire-Phelan cell to keep the complexity of the FE-models acceptable.

Foam cells are often elongated in one direction due to the foaming process during production. This elongation is quantified by the shape anisotropy $\lambda$ which is defined as the ratio of the length to the width of the cell. This quantity was determined on the same singe cell samples as before. The samples were rotated 4 times along the longitudinal axis over $90^{\circ}$ when measuring the length and width of the cells. This was done in order to average the irregular ellipsoidal shape of the cells and obtain a reliable value for the shape anisotropy. An average shape anisotropy of 1.41 with a standard deviation of 0.11 was found, which is in agreement to the values reported by Gong et al. [4]. 


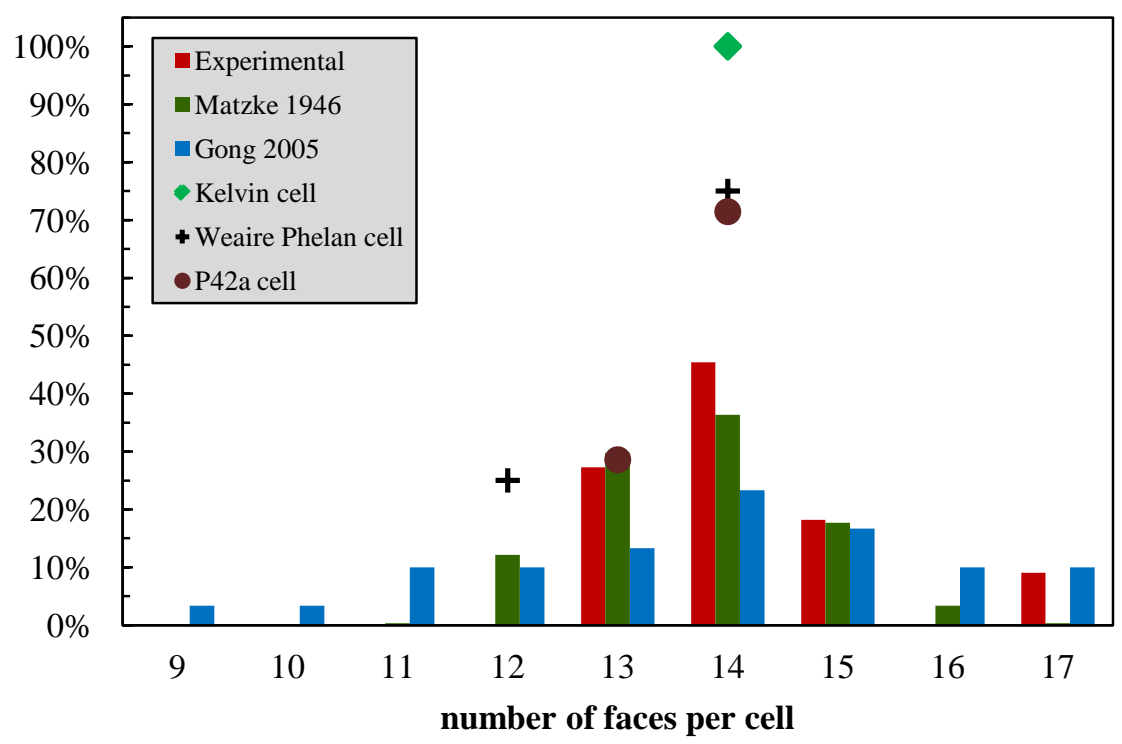

Figure 2: Distribution of the number of face per cell experimentally counted on 10 different single cell samples. Data from literature observations and 3 different unit cells are added to the figure as a comparison.
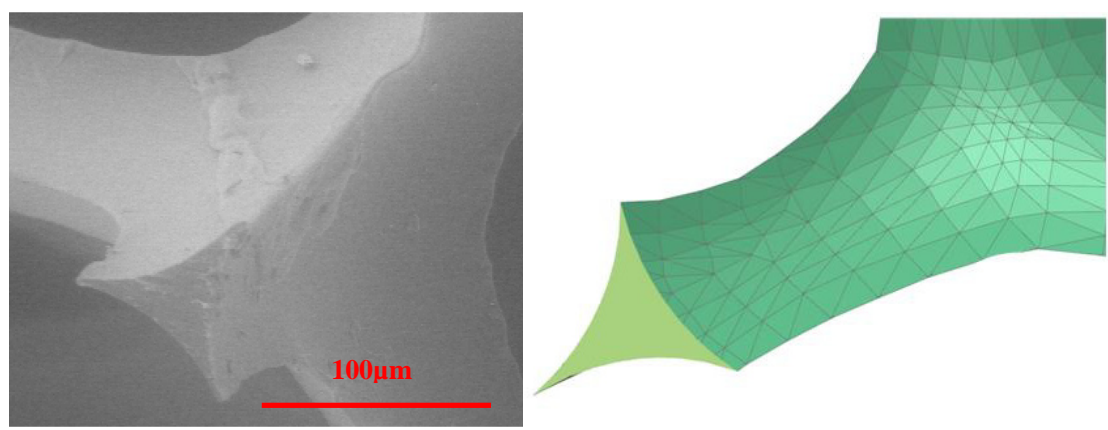

Figure 3: Scanning electron microscope image showing the typical plateau border cross sectional shape of the cell edges (left), Finite Element model of a cell edge with plateau border cross sectional shape (right). Notice the high degree of similarity between the FE-model and the SEM image.

Environmental Scanning Electron Microscopy was used to visualize the cellular structure of the open cell foam. The typical plateau border shape was observed in the cross section of the cell edges (fig. 3). 


\section{Experimental compression tests}

Compression tests in the rise direction of the open cell foam were performed in order to determine the elastic response of the material. All the samples had a square base and a height to width ratio of 2/3. Samples with a height of 20, 30, 45, 60 and 90mm were tested to determine the cell size effect. Moreover the first set of samples was cut with a knife while a saw was used for the second set. This distinction was made to investigate the influence of the sample damage on the stiffness response of the material due to the preparation method.

The sample size and damage effect was modelled using the size effects model developed by Onck et al. [12] In short, this model divides the sample into different volumes and appoints to each volume a local stiffness depending on the local boundary conditions. In this way the material in the centre of the sample will be linked to a higher stiffness compared to the material on the edge of the sample. Due to the unrestricted movement of the cell edges, the latter volume will actually get a zero-stiffness as it does not contribute to the mechanical response of the foam sample. More details on this model can be found in the relevant literature (Onck et al. [12], Andrews et al. [13]).

The foams were compressed with a constant strain rate of $1.6 .10^{-3} \mathrm{~s}^{-1}$ (ASTM standard [14]). All compression tests were repeated 10 times and statistically analysed with a p-value for significant difference of 0.05 .

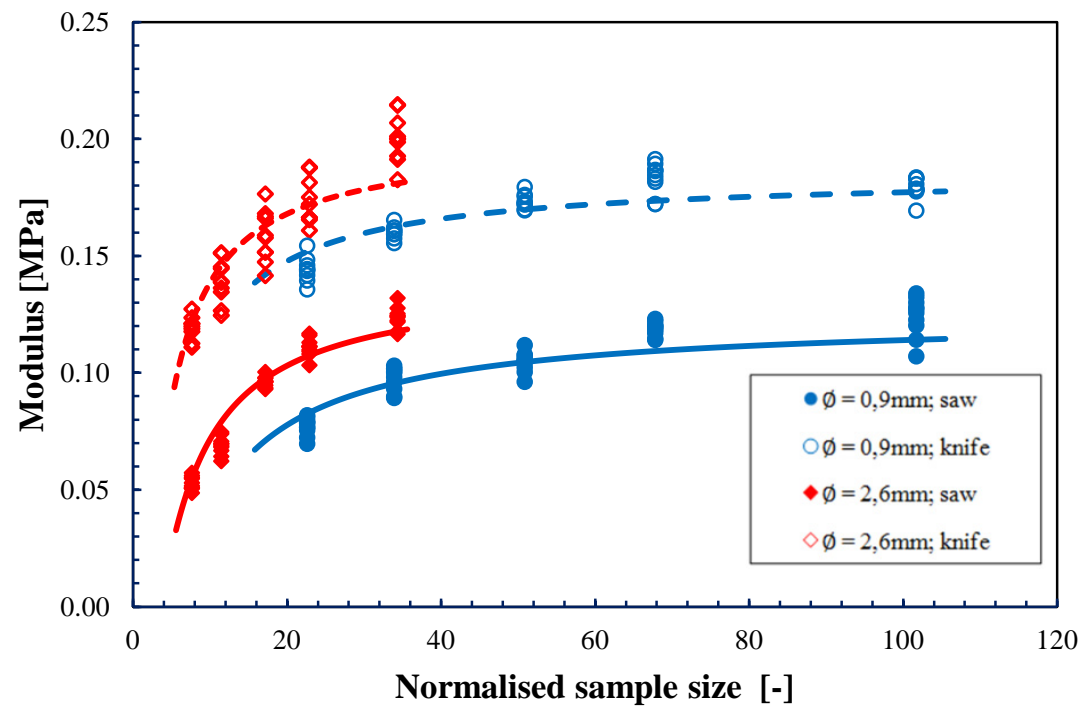

Figure 4: $\quad$ Flexible foam stiffness as a function of sample size and preparation method for two different cell diameters $(0.9$ and $2.6 \mathrm{~mm})$ of an open cell foam with a density of $24 \mathrm{~kg} / \mathrm{m}^{3}$.

The results of the compression tests are presented in figure 4 in which the stiffness of the open cell foam is expressed as a function of the sample height 
over cell size ratio. The stiffness of the foams clearly increases with increasing sample size up to a certain plateau value. This indicates a pronounced sample size effect. In contrast to the small cell size foam, the plateau value was not fully reached within the selected sample size range for the large cell open cell foam. Based on the obtained results, a sample size of at least 50 times the cell size is needed in order to assume no significant influence of the sample size on the stiffness. Moreover, the cell size has no significant influence on the stiffness of the foam. The noticeable differences between the cell sizes are actually caused by small differences in density. The experimental data also indicate the influence of the sample preparation method. It is clear that the samples which were prepared using a saw are a lot more damaged compared to the samples which were cut with a knife.

\section{Modelling of the open cell and complex foam}

\subsection{Building the FE-model}

In total three different FE-models were build: (1) a Kelvin cell FE-model with cylindrical cell edges as a first approximation, (2) a Kelvin cell FE-model in which the minimization of the surface energy determined the shape of the cell edges and (3) a Weaire-Phelan cell FE-model based on a similar surface energy minimisation.

In order to build the latter two models and homogenise their stiffness properties, different software package were linked through the scheme presented in figure 5.

\begin{tabular}{|c|c|c|c|}
\hline Create unit cell & $\begin{array}{c}\text { Transform } \\
\text { data }\end{array}$ & FE-Analysis & Process data \\
\hline $\begin{array}{l}\text { minimize the } \\
\text { surface energy } \\
\text { (Surface evolver) }\end{array}$ & $\begin{array}{c}\text { - Move nodes } \\
\text { on boundary } \\
\text { - Mirror } \\
\text { elements }\end{array}$ & $\begin{array}{l}\text { Apply periodic } \\
\text { boundary } \\
\text { conditions } \\
\text { (NX-Nastran) }\end{array}$ & $\begin{aligned}=> & \text { stiffness } \\
& \text { matrix } \\
= & >E_{\mathrm{i}}, v_{\mathrm{i}}, \mathrm{G}_{\mathrm{i}}\end{aligned}$ \\
\hline
\end{tabular}

Figure 5: Workflow for the construction of a Kelvin and Weaire-Phelan FE unit cell with periodic boundary conditions. The shape of the cell edges is completely governed by the surface energy minimisation within the surface evolver software.

The geometries of both unit cells are available within the standard surface evolver software (fig 6) (Brakke [15]). These files represent a dry closed cell foam which was transformed into a wet foam using the 'wetfoam.cmd' command (fig 6). Initially the cell edges of the wet foam are represented by nearly-equilateral triangular cross sections which replace the line edges of the 
dry foam. The wet foam structure was evolved by increasing the number of triangular facets that approximate the shape of the cell edges and using a sequence of surface energy minimising iterations (fig 6).
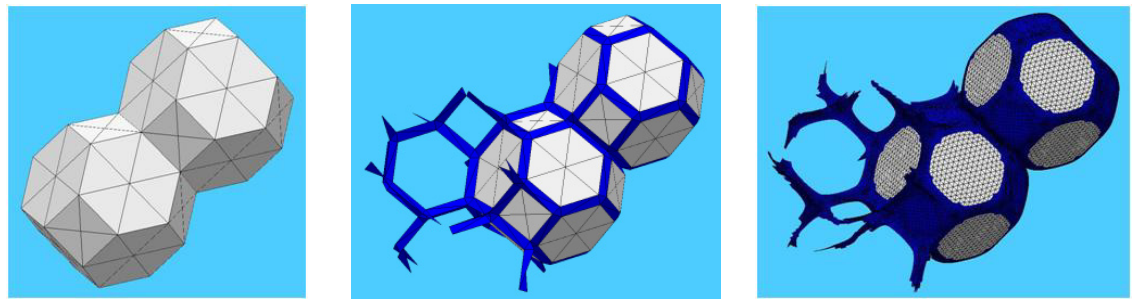

Figure 6: Two dry foam Kelvin cells build with standard surface evolver files (left), the same Kelvin cells transformed into a wet foam by adding material on the cell edges. These cell edges have a near-equilateral triangular cross sectional shape (middle), two Kelvin cells after minimsing the surface energy of the structure. The cross sectional shape of the cell edges is now the typical plateau border shape (right).

The obtained structure was exported as an ansys format file and processed via scripting. This was done in order to transform the surface evolver data into a readable FE-model for the Nastran solver. The transformation of the data includes the removal of the cell faces, repositioning of the nodes on the boundaries to comply with periodic boundary conditions and mirror and rotate the nodes and elements to construct a complete unit cell out of $1 / 8$ of the unit cell (fig 7). In order to investigate the influence of anisotropy $\lambda$, different models were elongated in one direction (fig. 7).
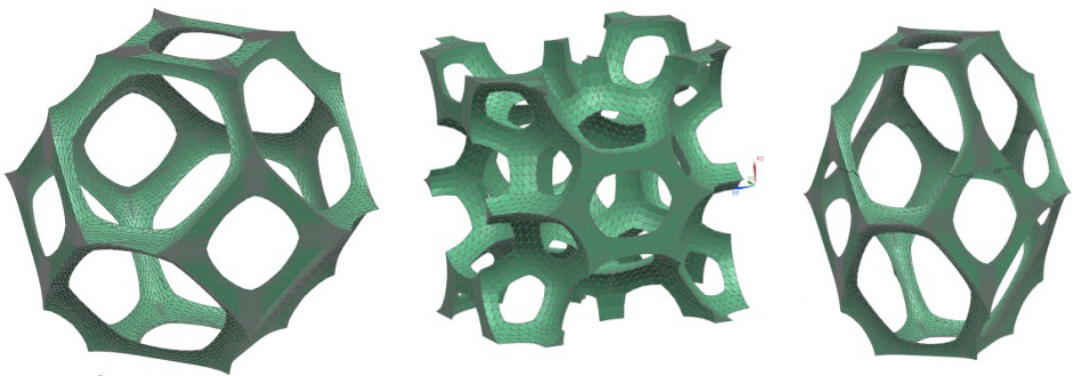

Figure 7: $\quad$ FE-model of the Kelvin cell unit cell with $\lambda=1$ and relative density $=2 \%$ (left), FE-model of the Weaire-Phelan unit cell with $\lambda=1$ and relative density $=6 \%$ (middle), FE-model of the Kelvin cell unit cell with $\lambda=1,4$ and relative density $=2 \%$ (right). All models consist out of quadratic tetragonal elements. 
Quadratic tetragonal elements were used to build a full 3D model of the unit cells. The FE-models were loaded with periodic boundary conditions within the Siemens-NX software and solved with a Nastran solver. Asymptotic homogenisation was used to reconstruct the stiffness tensor of the cellular structure. The engineering constants were derived out of this stiffness tensor.

\subsection{Results and discussion}

The influence of the relative density on the elastic material properties of the open cell foam is presented in figures 8,9 and 10 . A solid material stiffness of $69 \mathrm{MPa}$ and poisson coefficient of 0.4 was used for all FE-models (Gong et al. [4]).

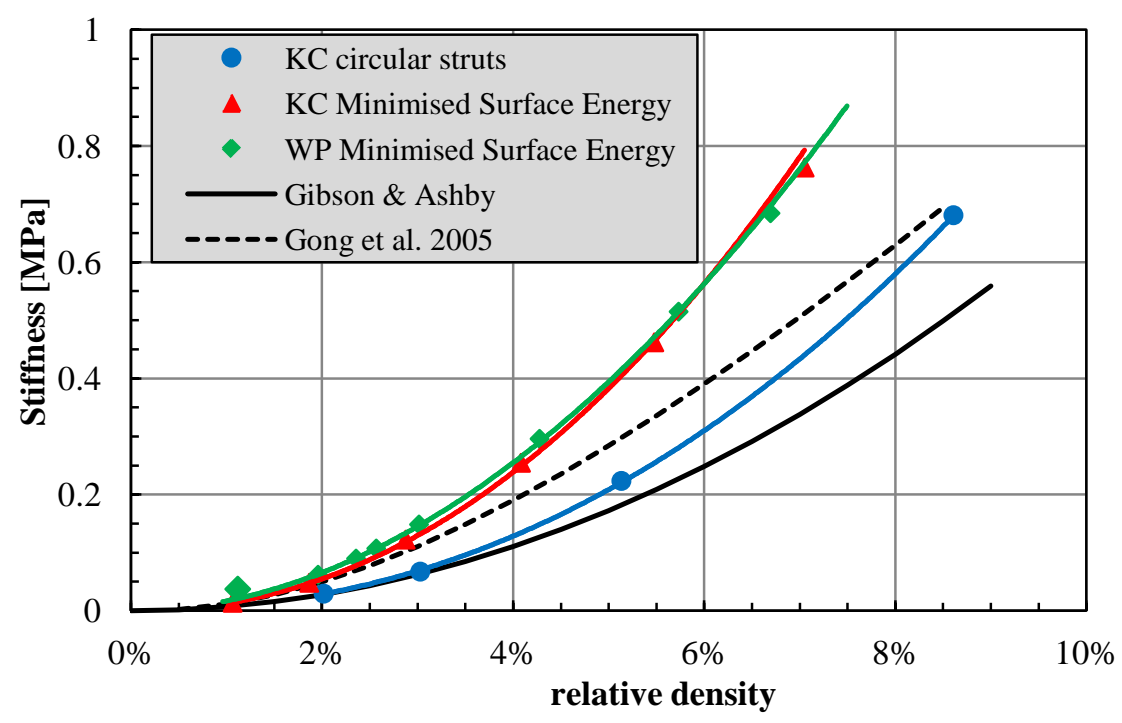

Figure 8: Influence of relative density on the stiffness of open cell foams with an anisotropy of 1 modelled by different FE-models and compared against the analytical models of Gibson and Ashby [10] and Gong et al. [4].

Figure 8 indicates that both models with a minimised surface energy possess a similar dependence of stiffness on the density of the foam. When the open cell foam is modelled as a Kelvin cell with a circular cross sectional shape of the cell edges, the structure behaves significantly less stiff. This can be explained by the bending dominated deformation mechanism of the open cell. Since the moment of inertia of a plateau border shape is $70 \%$ higher compared to a circle with identical surface area, the stiffer response of the minimal surface energy models was expected. The model of Gibson and Ashby [10] is based on a simple cubical unit cell and classical beam theory to determine the stiffness of a cellular structure. This model uses a high degree of simplification which results in a 
lower bound for the other models. The model of Gong et al. [4] is based on a Kelvin cell geometry with plateau border shaped cell edges. Moreover the nonconstant cross sectional area of the cell edges and the shear deformation was taken into account. However the additional material in the nodes of cell edges was not taken into account. This results in an intermediate stiffness as a function of the foam density.

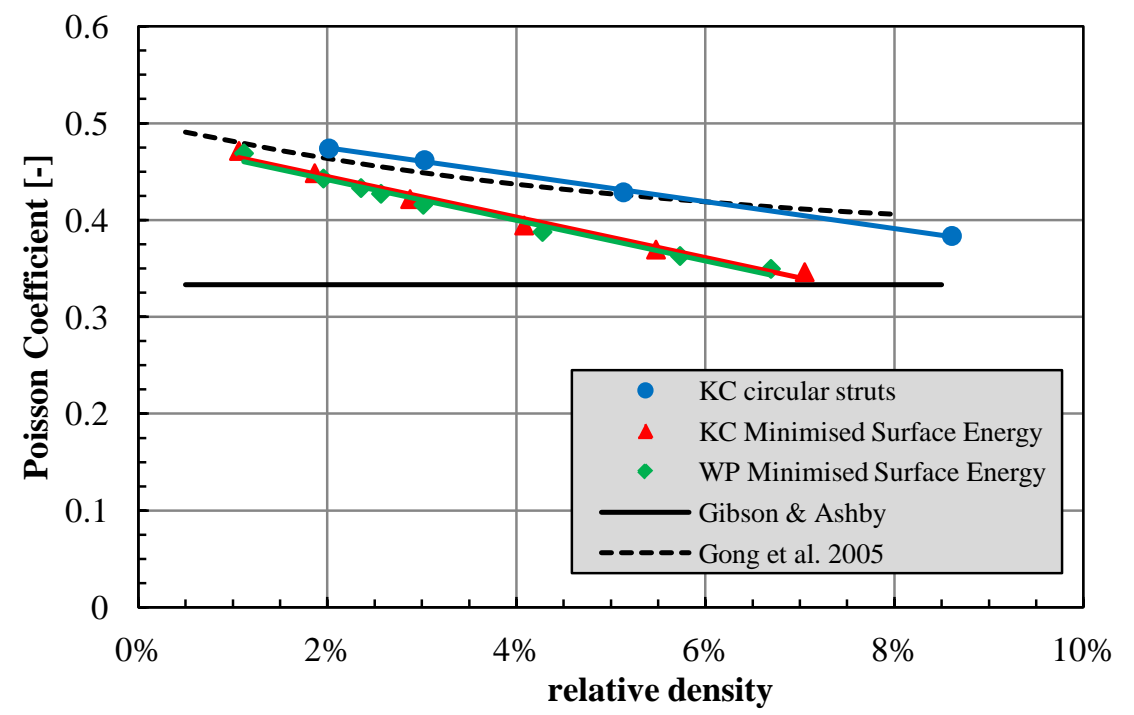

Figure 9: Influence of relative density on the poisson coefficient of open cell foams with an anisotropy of 1 modelled by different FE-models and compared against the analytical models of Gibson and Ashby [10] and Gong [4].

Figure 9 presents the dependence of the poisson coefficient on the relative density. In agreement to the stiffness results in figure 8 , both models with a minimised surface energy show a similar behaviour. Except for the results of Gibson and Ashby all models show a decreasing poisson coefficient with increasing foam density. The poisson ratio value of 1/3 suggested by Gibson and Ashby is based on a linear elastic isotropic assumption and partially on experimental data. The former is however not necessarily the case for open cell polyurethane foams. The FE-models with minimised surface energy seem to predict a stronger dependency of the poisson ratio on density than the nearly coinciding analytical model of Gong et al. [4] and the FE-model with circular cell edges. The higher the ratio of bending to tensile stiffness of the cell edges is, the steeper the decrease in poisson ratio will be (Zhu et al. [17]). This is confirmed by the comparison between the FE-models with circular cell edges 
and the minimised surface energy FE-models with plateau border shaped cell edges.

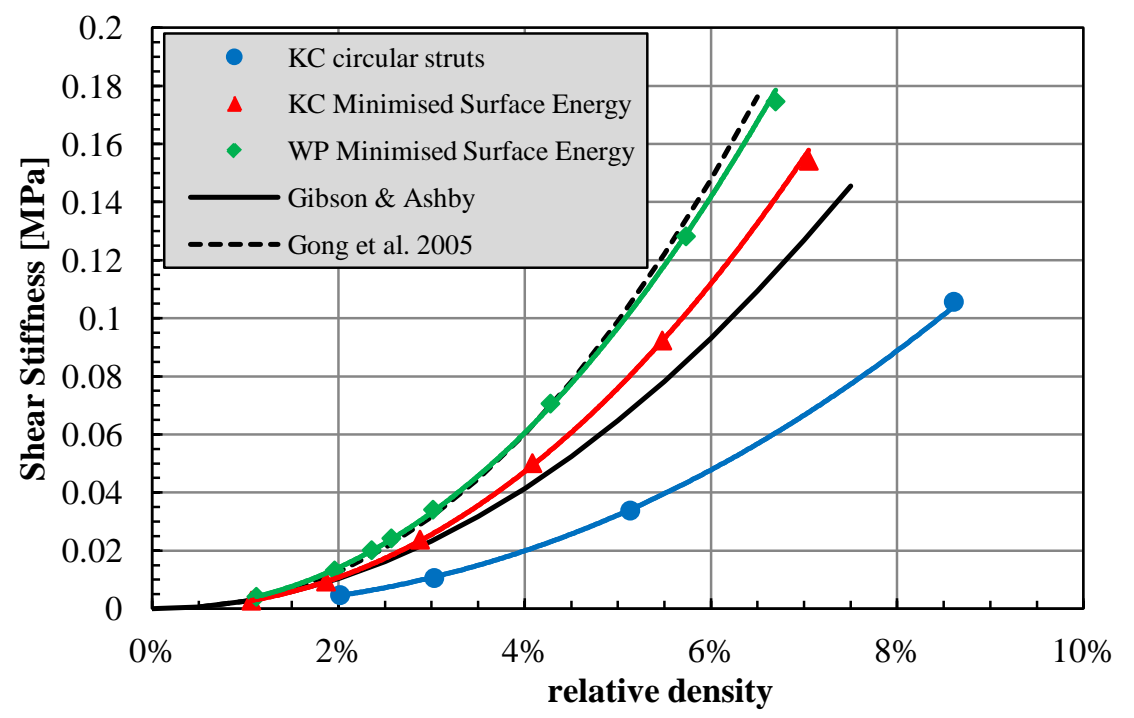

Figure 10: Influence of relative density on the shear stiffness of open cell foams with an anisotropy of 1 modelled by different FE-models and compared to the analytical models of Gibson and Ashby [10] and Gong et al. [4].

As in the case of the stiffness, the dependency of the shear stiffness on the relative density can be approximated by a power law relationship. The shear stiffness of the different FE-models increases with increasing similarity of the unit cell to a real foam topology. In this way the minimal surface energy model of the Weaire-Phelan unit cell predicts the stiffest response and agrees with the analytical model of Gong et al. [4].

The influence of the anisotropy of the foam cells on the stiffness is presented in figure 11. A linearly increase in stiffness is observed in the direction of the elongated cells while a decrease is observed in the direction perpendicular to it. Both FE-models with a minimised surface energy predict a similar decrease in stiffness. The Weaire-Phelan model however, predicts a larger increase in stiffness. The FE-results are compared to the model proposed by Huber and Gibson [16]. This model is based on the rudimental unit cell of Gibson and Ashby [10] and should therefore be used as a first approximating model. Regarding the experimentally determined real foam anisotropy of 1.4, the increase of the stiffness in the elongated direction is 70 to $90 \%$ compared to a foam with an anisotropy of 1 with the same density. The decrease in stiffness in the direction perpendicular to it is 30\% for both FE-models. 

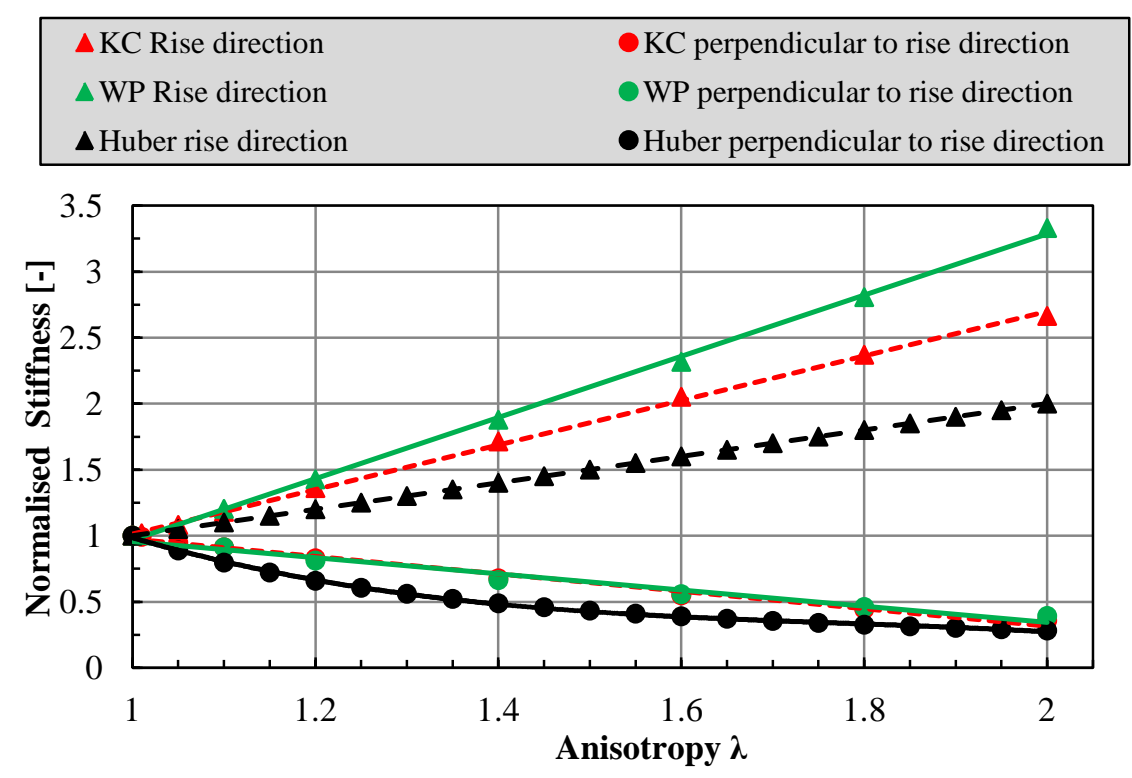

Figure 11: Influence of anisotropy $\lambda$ on the stiffness of an open cell foam modelled by different FE-models compared against the analytical model of Huber [16]. The relative density of the foam is $2 \%$.

\section{Conclusions}

Within this study the elastic behaviour of an open cell polyurethane foam was analysed. This was done by quantifying the morphology of the cellular structure, experimental compression tests and FE-modelling.

Optical and scanning electron microscopy proved to be very useful to visualise and analyse the cellular morphology. The number of edges per face and number of faces per cell was determined and compared to literature and different possible FE-unit cells. The classical Kelvin cell and more complex WeairePhelan cell structures were selected to be used as a unit cells for the FE-models. The main advantage of the Weaire-Phelan cell is the presence of pentagonal faces which agrees with experimental observations and meets the absence of them in the Kelvin cell structure.

Experimental compression tests on two flexible open cell polyurethane foams with identical density and different cell size revealed a pronounced sample size and damage effect. This was taken into account, using the sample size effects model of Onck et al. [12]. As a conclusion sample size and preparation method should be thoroughly considered in advance.

The use of surface evolver allows us to construct FE-models with a remarkable similarity to real foam topologies. The dependence of elastic properties of open cell foams on relative density and anisotropy was investigated 
and agreed with known relationships in literature. The stiffness and poisson ratio predictions as a function of density of the Weaire-Phelan topology nearly coincide with the Kelvin cell results. However, the Weaire-Phelan based FEmodel indicates a higher sensitivity of the shear stiffness on the foam density and of the stiffness on the anisotropy value.

Therefore, it should be noted that the topology of the FE-models should match the topology of real foam structures as good as possible. The use of completely random foam FE-structures is however not advisable because of the large computational power which would be needed. The models with minimised surface energy, presented in this paper, strike the golden mean between a high degree of similarity to a real open cell foam and the need for low computational effort.

\section{References}

[1] Randall, D. and Lee, S., The polyurethanes book, John Wiley and sons LTD: Trento Italy, 2010.

[2] Mills, N., Polymer Foams Handbook, Butterworth-Heineman: Great Britain, 2007.

[3] Matzke, E. B. The three-dimensional shape of bubbles in foam - an analysis of the role of surfaces in three-dimensional cell shape determination. American journal of Botany 33, pp. 58-80, 1946.

[4] Gong, L., Kyriakides, S. and Jang, W.-Y., Compressive response of opencell foams. Part I: Morphology and elastic properties. International Journal of solids and structures, 42, pp. 1355-1379, 2005.

[5] Mills, N.J., The high strain mechanical response of the wet Kelvin model for open-cell foams, International Journal of solids and structures, 44, pp. 51-65 2007.

[6] Jang, W.-Y., Kraynik, A. and Kyriakides, S., On the microstructure of open-cell foams and its effects on elastic properties. International Journal of solids and structures, 45, pp. 1845-1875, 2008.

[7] Wismans, J.G.F., Van Dommelen, J.A.W., Govaert, L.E. and Meijer, H.E.H., CT-based Modelling of Structured Polymers. Journal of cellular Plastics, 45, pp. 157-179, 2009.

[8] Phelan, R., Weaire, D. and Brakke, K., Computation of Equilibrium Foam Structures Using the Surface Evolver. Experimental Mathematics, 4(3), pp. 181-191, 1995.

[9] Gabbrielli, R., A new counter-example to Kelvin's Conjecture on minimal surfaces. Philosophical Magazine Letters, 89(8), pp. 483-491, 2009.

[10] Gibson, L.J., and Ashby, M.F., Cellular Solids, Cambridge University Press: Cambridge United Kingdom, 1997.

[11] Warren, W.E. and Kraynik, A.M., Linear Elastic Behaviour of a LowDensity Kelvin Foam with Open Cells. Journal of Applied Mechanics, 64, pp. 787-794, 1997. 
[12] Onck, P., Andrews, E.W., Gibson, L.J. Size Effects in Ductile Cellular Solids. Part I: Modeling. International. Journal of Mechanical Sciences, 43, pp. 681-699, 2001.

[13] Andrews. E.W., Gioux. G., Onck. P., Gibson. L.J. Size Effects in Ductile Cellular Solids. Part II: Experimental Results. International Journal of Mechanical Sciences, 43, pp. 701-713 2001.

[14] ASTM D 1621-04a. Std. test method for compressive properties of rigid cellular plastic. 2004.

[15] Brakke, K.A., The Surface evolver. Experimental Mathematics 1, pp. 141165, 1992. available from http://www.susqu.edu/facstaff/b/brakke/evolver/.

[16] Huber, A.T., Gibson, L.J., Anisotropy of foams, Journal of materials science, 23, pp. 3031-3040, 1998.

[17] Zhu, H.X., Knott, J.F., Mills, N.J., Analysis of the elastic properties of open-cell foams with tetrakaidecahedral cells, Journal of the mechanics and physics of solids, 45(3), pp. 319-343, 1997. 